\title{
Erratum to: A threshold method for immunological correlates of protection
}

\author{
Xuan Chen ${ }^{1}$, Fabrice Bailleux ${ }^{2}$, Kamal Desai ${ }^{3 *}$, Li Qin $^{4,5}$ and Andrew J. Dunning ${ }^{6}$
}

\section{Erratum}

After publication of the original article [1], it came to the authors' attention that there was an error in the Testing for the existence of a threshold sub-section of the Methods.

Two formulae originally published in this sub-section were incorrect; the correct expressions are the negatives of the expressions published. The formulae should have appeared as follows:

$$
\mathrm{D}=2 \mathrm{l}(\mathrm{a}, \mathrm{b}, \mathrm{\tau})-2 \mathrm{l}\left(\mathrm{a}^{\prime}\right)
$$

and

$$
D^{\prime}=2 l(a, b, \tau)-2 l\left(a^{\prime}\right) \text { for } a>b .
$$

\section{Author details}

'Sanofi Pasteur, Beijing, China. ${ }^{2}$ Sanofi Pasteur, Marcy L'Etoile, France. ${ }^{3}$ United Biosource Corporation, London, UK. ${ }^{4}$ Statistical Center for HIV/AIDS Research and Prevention, Fred Hutchinson Cancer Research Center, Seattle, WA, USA.

${ }^{5}$ Present address: Amazon.com, Inc, Seattle, WA, USA. 'Sanofi Pasteur,

Swiftwater, PA, USA.

Published online: 02 February 2017

\section{Reference}

1. Chen X, Bailleux F, Desai K, Qin L, Dunning AJ. A threshold method for

immunological correlates of protection. BMC Med Res Methodol. 2013;13:29. doi:10.1186/1471-2288-13-29. 